\title{
Effect of iguratimod on diclofenac metabolism by CYP2C9 in rats and human recombinant CYP2C9 yeast cells
}

\author{
Muhammad Usman', Zhu Zhen-Han', Chang Ze-Na1, Han Jun-Ping', Qian Wen², \\ Yang Chang-Qing ${ }^{1 *}$, Nishikawa Miyu ${ }^{3}$, Sakaki Toshiyuki ${ }^{3}$
}

\begin{abstract}
${ }^{1}$ School of Basic Medicine and Clinical Pharmacy, China Pharmaceutical University, Nanjing, China, ${ }^{2}$ Nanjing BRT-Biomed Co. Ltd. Nanjing, China, ${ }^{3}$ Department of Biotechnology, Faculty of Engineering, Toyama Prefectural University, Imizu,
\end{abstract} Toyama, Japan

\begin{abstract}
Iguratimod (IGU, also known as T-614), a novel disease modifying antirheumatic drug intended to cure patients with rheumatoid arthritis (RA). The purpose of this study is to evaluate the effect of IGU on the pharmacokinetics of CYP2C9 probe drug diclofenac and its metabolite 4'-hydroxy diclofenac in vivo and in vitro. In in vivo experiments, 24 rats were randomly assigned to three groups consisting of the control group (Normal saline), low dose IGU group (10 mg/kg) and high dose IGU group (30 $\mathrm{mg} /$ $\mathrm{kg}$ ). Blood samples were collected from orbital sinuses vein before 1 hour and serial times of giving diclofenac $(15 \mathrm{mg} / \mathrm{kg})$ to all the rats. Plasma concentration of diclofenac and its metabolite $4^{\prime}$-hydroxy diclofenac were assayed by high performance liquid chromatography. Pharmacokinetic parameters were assessed by Winnonlin 6.4 pharmacokinetic software. Moreover, in vitro studies were performed in recombinant human CYP2C9 yeast cell system. IGU at low dose showed no significant differences in the pharmacokinetic parameters of diclofenac and 4-hydroxy diclofenac in vivo when compared with control group $(p>0.005)$. However, at the high dose of IGU, the pharmacokinetic parameters of 4 ' -hydroxy metabolite of diclofenac increase in half-life $\left(\mathrm{T}_{1 / 2}\right)$ and mean area under the curve $\left(\mathrm{AUC}_{0 \rightarrow 24}\right)$, while a decrease in mean clearance $(\mathrm{CL}, \mathrm{mL} / \mathrm{h} / \mathrm{kg})$ and volume of distribution $\mathrm{Vz}(\mathrm{mL} / \mathrm{kg})$. In addition, in in vitro study, high doses of IGU reduces the metabolism rate of diclofenac. IGU at high dose significantly increase the pharmacokinetics parameters of 4'-hydroxy diclofenac in rats. Additionally, it also showed the potent inhibitory effect on diclofenac metabolism in recombinant human CYP2C9 yeast cells.
\end{abstract}

Keywords: Iguratimod/effects. Diclofenac/effects. 4-hydroxy diclofenac. CYP2C9/pharmacokinetics. Inhibitory.

\section{INTRODUCTION}

Iguratimod (IGU or T614), a novel diseasemodifying antirheumatic drug (DMARD), used solely in Japan and China for the tratment of rheumatoid arthritis (RA). It is noteworthy that IGU shows a useful effect in RA patients with inadequate response to methotrexate (Ishiguro et al., 2013; Hara et al., 2014). After numerous oral doses of the IGU in healthy male subjects $(25 \mathrm{mg}$ for 14 days), several metabolites were identified in the plasma, including M1 (deformaylated form), M2 (N-acetylated form), M3, M4 and M5 (6- phenoxy- hydroxylated form),

\footnotetext{
*Correspondence: Y. Chang-Qing. School of Basic Medicine and Clinical Pharmacy, China Pharmaceutical University, Nanjing 211198, China. Phone: +8615005179447. SAKAKI Toshiyuki; (yang670315@hotmail.com) (iD)
}

of which M1 and M2 were recognized to be effective metabolites. IGU is metabolized by cytochrome P450 (CYP) which results in the formation of M1 metabolite, irrespective of recombinant CYP isoforms studied in the absence or presence of reduced nicotinamide adenine dinucleotide phosphate (NADPH). Previous studies have suggested that the metabolism of M1 through $N$-acetyl transferase results in the formation of M2 metabolite, while M4 and/or M5 metabolites are formed from IGU metabolism by several other isoforms including CYP2C9, CYP1A2, and CYP3A4 (Yamaori et al., 2015). When manufacturing approval of IGU was granted in Japan, the directions specified that caution must be considered for the combined use of IGU with warfarin, established on the statement that IGU extended prothrombin time in rat's co-administered warfarin. Subsequently, the benefit 
of the IGU in combination with warfarin has changed from precaution to contraindication.

The health society of Japan studied in vitro inhibitory effect of the IGU in human liver microsomes (HLMs), which demonstrated that at $100 \mu \mathrm{M}$ concentration the activity of IGU is inhibited by $50 \%$, as described in a published report (Ruddy, Austen, 1975) (4). Owing to the inhibitory effect and maximum uncontrolled hepatic response concentration, the probability of interaction among IGU and CYP2C9 substrate drugs is inadequate as estimated. A previous report from Toyama Chemical $\mathrm{CO}$, Ltd demonstrated that the inhibitory effect of CYP2C9 inhibitors varied depending on the substrate used. In this above study, tolbutamide used as a probe substrate for CYP2C9 (Hanatani et al., 2003; Kumar et al., 2006). Astonishingly, there was a 5-fold increase in $\mathrm{Ki}$ values for tolbutamide 4-hydroxylation than S-warfarin 7-hydroxylation, confirmed on one third of CYP2C9 inhibitors (Kumar et al., 2006). Thus, IGU showed inhibitory effect on CYP2C9, may vary between S-warfarin and tolbutamide.

Diclofenac (2-[2-(2,6-dichlorophenylamino) phenyl] acetic acid) is a broadly used non-steroidal antiinflammatory drug (NSAID) specified for the treatment of irritation, RA, osteoarthritis, ankylosing spondylitis and discomfort in circumstances such as stiffness and severe muscle pain (Small, 1989). Diclofenac is metabolized through oxidative biotransformation and glucuronidation in humans. Oxidative biotransformation of the aromatic ring is performed through cytochrome $\mathrm{P} 450$ and hydroxylation of the dichlorophenyl group is particularly catalyzed by CYP2C9 to form 4'-hydroxy diclofenac as the main metabolite, while glucuronidation of the carboxylic acid unit is mediated through uridine diphosphate glucuronosyltransferase (UGT) 2B7 (Leemann, Transon, Dayer, 1993). The CYP2C9 enzyme is responsible for 3 ' and 4'-hydroxylation, whereas CYP2C and CYP3A4 enzymes mediate through 5'-hydroxylation (Leemann, Transon, Dayer, 1993; Bort et al., 1999) (Figure 1). Oxidation of numerous drugs i.e. diclofenac, flurbiprofen, warfarin, hexobarbital, phenytoin, and tolbutamide are structurally diverse substrates used recently, and their metabolism occurs mainly through CYP2C9 enzyme that is abundant in human liver microsomes (HLMs) (Goldstein, de Morais, 1994; Tracy et al., 1996; Inoue et al., 1997; Miners, Birkett, 1998; Yamazaki et al., 1998). Diclofenac is used as the substrate for studies of CYP2C9 metabolic activity frequently because 4 '-hydroxy diclofenac is metabolized through CYP2C9 (Mancy et al., 1999).

The aim of our study is to examine and analyse the effect of IGU on the metabolism of diclofenac in rats

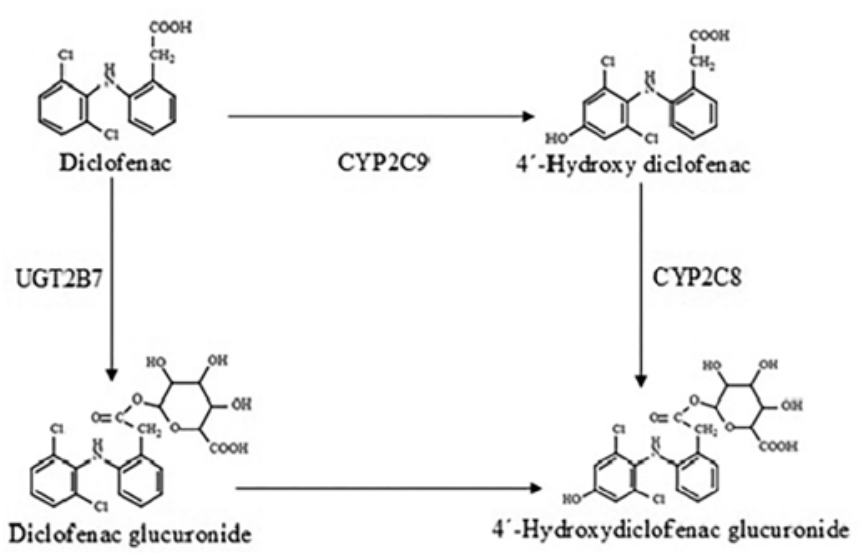

FIGURE 1 - Proposed primary metabolic pathways for diclofenac in humans.

and estimate the diclofenac and 4-hydroxy diclofenac concentration in plasma by HPLC. In addition, we also investigate and explore the effect of IGU on human recombinant CYP2C9 yeast cells. We also elucidate the potential interaction between IGU and the CYP2C9 substrate. The potential of in vitro interaction between IGU and UGT enzyme inhibition is additionally discussed.

\section{MATERIAL}

\section{Instruments}

LC-2010AHT High Performance Liquid Chromatography (Shimadzu Co., Japan), a quaternary high-pressure pump, DAD UV detector, SHIMADZU Lab Solutions LC solution workstation.

\section{Reagents}

Acetonitrile of HPLC grade purchased from Merck KGaA, (Germany). Trifluoroacetic acid (purity: $99 \%$, batch number 150429824D). Bifendate reference standard (China Institute of pharmaceutical and biological products, purity: $99 \%$, batch number: 100192-200503. 4'-hydroxy-diclofenac reference standard (Nanjing BRTBiomed Co. Ltd. batch number 160105, purity: 99\%). Diclofenac injection (Furen Pharmaceutical Group Co., Ltd. batch number 1404203, specification: $50 \mathrm{mg} / 2 \mathrm{ml}$ ). IGU (Xiansheng Co., Ltd. Batch number 42-160201, specification: $25 \mathrm{mg}$ )

\section{Animals}

Several experimental trials were performed on 24 male Sprague-Dawley (S.D.) rats, provided by the 
Shanghai Jie Esprit experimental animal Co. License number: SCXK 2013-0006 (Shanghai, China), weighing approximately in the range of $180-220 \mathrm{~g}$. The twenty four rats were randomly divided into three groups (4 per cage) under normal lighting, temperature $\left(25 \pm 2^{\circ} \mathrm{C}\right)$ and moisture. Afterward, rats from each group of rats was fasted overnight with free access to water and pellet food. The study was favored by the animal control and handling Agency of China Pharmaceutical University and was presented in agreement through the national institute of health guidelines for the control and handling of animals.

\section{METHOD}

\section{Pharmacokinetic study of diclofenac and 4-hydroxy diclofenac in rats}

\section{Experimental procedure}

Twenty-four S.D. rats were randomly divided into three groups of eight animals in each group i.e. control group (normal saline $0.9 \%$ ); low dose IGU group (10mg/ $\mathrm{kg}$, p.o); high dose IGU group (30mg/kg p.o), rats were adaptive feeding for one week. Meanwhile, appropriate medicinal dose was administered twice a day for seven consecutive days. In this part of the experiment 1 hour before collecting of blood sample, diclofenac $15 \mathrm{mg} / \mathrm{kg} i v$ bolus was given. Then the blood samples were collected in $2 \mathrm{~mL}$ Eppendorf tubes containing sodium citrate as anticoagulant before and after diclofenac administration at $0.05,0.15,0.30,0.45,1,1.5,2,3,4,6,8,10,12$ and 24 $\mathrm{h}$ from orbital sinuses vein. Plasma separated by $5000 \mathrm{rpm}$ centrifugation for $10 \mathrm{~min}$ at $4{ }^{\circ} \mathrm{C}$, and stored at $-20^{\circ} \mathrm{C}$ deep freezer until further experiments. HPLC technique was used to examine the plasma concentration of diclofenac.

\section{Determination of diclofenac plasma concentration by HPLC method}

\section{Plasma sample preparation}

Approximately $50 \mu \mathrm{L}$ plasma was added to a $1.5 \mathrm{~mL}$ centrifuge tube, followed by $25 \mu \mathrm{L}(40 \mathrm{mg} / \mathrm{L})$ of internal standard Bifendate, $25 \mu \mathrm{L}$ of acetonitrile was combined and mixed for $2 \mathrm{~min}$ in a vortex mixer and centrifuged at $12000 \mathrm{rpm}, 4^{\circ} \mathrm{C}$ for $10 \mathrm{~min}$. After this centrifuge mixture, take out the supernatant for HPLC analysis.

\section{Chromatographic conditions}

The chromatographic column expended for this analysis was Galaksil EF-C18H (250mm x 4.6mm, $5 \mu \mathrm{m})$.
The mobile phase is consist of acetonitrile: water ( $55: 45$, add $0.1 \%$ trifluoroacetic acid). The injection volume was $10 \mu \mathrm{L}$. The flow rate was controlled to $1.0 \mathrm{ml} / \mathrm{min}$ and the emission was observed at $280 \mathrm{~nm}$. The entire run time of the system was fixed at $15 \mathrm{~min}$.

\section{Preparation of stock solutions}

Standard solutions of $1 \mathrm{mg} / \mathrm{mL}$ of diclofenac and 4 '-hydroxy-diclofenac were made distinctly in methanol and kept at $4{ }^{\circ} \mathrm{C}$. Working standard solution were made by successively mitigating the stock solution using acetonitrile. The internal standard stock solution (bifendate, $40 \mu \mathrm{g} / \mathrm{mL}$ ) was made in acetonitrile. Calibration samples were made by adding mixtures of the standard combination with blank rat plasma to develop a concentration series of $0.25,0.5,1,2,5,10,20$ and 40 $\mu \mathrm{g} / \mathrm{mL}$ for diclofenac and 4 '-hydroxy diclofenac. Quality control samples of diclofenac and 4'-hydroxy diclofenac in different concentration were similarly organized in a related way. All solutions were kept at $-20^{\circ} \mathrm{C}$ previously consume. All samples remained collected at intervals for diclofenac bioanalysis and were stored frozen at $-80^{\circ} \mathrm{C}$ until examined.

\section{Extraction technique}

From plasma, the drug was obtained with acetonitrile. In a $1.5 \mathrm{~mL}$ centrifuge tube, $50 \mu \mathrm{L}$ of plasma was put in. The plasma was triggered by the adjunct of $25 \mu 1$ of acetonitrile, and $25 \mu \mathrm{L}$ of bifendate internal standard, and then the tube was vortexed for $2 \mathrm{~min}$ and centrifuged at $12000 \mathrm{rpm}$, $4^{\circ} \mathrm{C}$ for $10 \mathrm{~min}$. The supernatant was moved to a sterile, similarly marked tube. The ensued mixture was vaporized, and reorganized with the mobile phase. From this sample, $10 \mu 1$ was injected to HPLC.

\section{Construction of calibration curve}

The calibration curve was assembled using highest area against minimal concentration. According to diclofenac, 4'-hydroxy diclofenac and internal standard peak area ratio $(\mathrm{y})$ of concentration $(\mathrm{x})$ linear regression analysis was achieved respectively. The grade of the area controlled by the scheme of least-square regression assay exploited to estimate the diclofenac and $4^{\prime}$-hydroxyl diclofenac concentration in the unidentified sample.

\section{Determination of diclofenac metabolism rate in recombinant human CYP2C9 yeast cells by HPLC}

The recombinant $\mathrm{S}$. cerevisiae cells expressing human P450s were cultured in artificial minimal medium 
comprising $40 \%$ glucose, sterile distilled water, $1 \mathrm{M}$ potassium Phosphate Buffer ( $\mathrm{pH} 7.4$ ), and then low, medium and high doses of IGU were added to the CYP2C9 yeast cells culture reaction system. When the cell concentration of culture reached $100 \mathrm{mM} / \mathrm{L}, 2 \mathrm{~h}$ before diclofenac in dimethyl sulfoxide (DMSO) was adjoined to the system at a final concentration of $1000 \mu \mathrm{M}$. At $24 \mathrm{~h}$ after addition of diclofenac, $100 \mu \mathrm{L}$ of the culture were obtained with $200 \mu \mathrm{L}$ of methanol and centrifuged at $12000 \times \mathrm{g}$ for $10 \mathrm{~min}$. The subsequent filtrate was solubilized with methanol and operated to HPLC under the subsequent circumstances: column, Galaksil EF-C18H (250mm x $4.6 \mathrm{~mm}, 5 \mu \mathrm{m})$; UV detection, $280 \mathrm{~nm}$; flow rate, $1.0 \mathrm{~mL} / \mathrm{min}$; column temperature, $40{ }^{\circ} \mathrm{C}$; injection volume, $10 \mu \mathrm{L}$; and the mobile phase consisted of water: acetonitrile (containing 0.1\% trifluoroacetic acid) at 45:55. The inhibitory response rate of diclofenac metabolism in human recombinant CYP2C9 yeast cells were calculated using the formula.

Inhibitory response rate $=\frac{C_{\text {Diclofenac }}(0 h)-C_{\text {Diclofenac }}(24 h)}{C_{\text {Diclofenac }}(0 h)} \times 100 \%$

\section{Statistical analysis}

All statistics were calculated using IBM SPSS statistics version 22. The PK parameters were calculated using WinNonlin non-compartmental model (Pharsight Co, Mountain View, CA, and the USA. 6.0). The PK parameters of diclofenac and 4-hydroxy diclofenac for
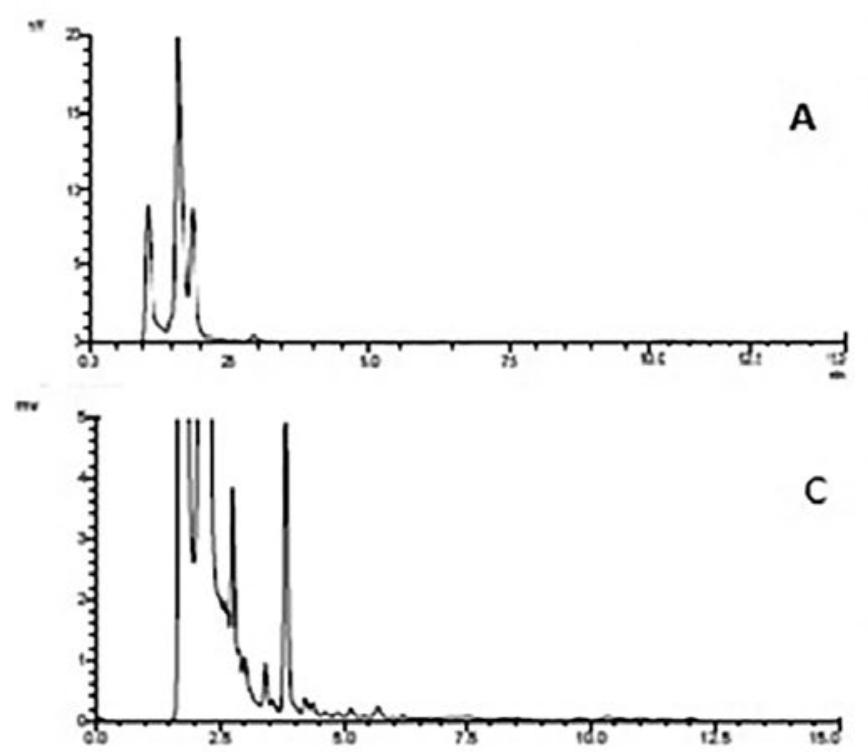

groups were compared by means of one-way ANOVA pursued by Games-Howell Post Hoc test. The in vitro inhibitory study for diclofenac were also analyzed by the same test above. The $p<0.05$ were considered significant.

\section{RESULTS}

\section{Assessment of diclofenac and 4-hydroxy diclofenac in plasma samples by HPLC}

By analyzing blank plasma samples the selectivity of HPLC analysis was studied, which did not display any interfering components (Figure 2A). The entire run time of the technique was established at $15 \mathrm{~min}$. Retention time was $11.26 \mathrm{~min}$ and $5.22 \mathrm{~min}$ for diclofenac and 4 '-hydroxy diclofenac respectively (Figure 2).

The linear regression analysis of diclofenac and $4^{\prime}$-hydroxy diclofenac was formed by calculating the peak-area ratio of diclofenac, $4^{\prime}$-hydroxy diclofenac and internal standard. The method displays excellent linearity over a concentration range of $0.25-40 \mu \mathrm{g} . \mathrm{mL}^{-1}$ for both diclofenac and $4^{\prime}$-hydroxy diclofenac $(\mathrm{y}=0.1083 \mathrm{x}+$ $0.0014, \mathrm{r}=0.999, \mathrm{n}=6 ; \mathrm{y}=0.043 \mathrm{x}+0.0013, \mathrm{r}=0.999$, $\mathrm{n}=6$ ) respectively. The limit of detection for diclofenac and 4 '-hydroxy diclofenac was $0.15 \mu \mathrm{g} \cdot \mathrm{mL}^{-1}$.

In accordance with the method of preparation containing diclofenac $(0.5,10,40 \mu \mathrm{g} / \mathrm{mL})$ and $4^{\prime}$-hydroxy diclofenac $(0.5,10,40 \mu \mathrm{g} / \mathrm{mL})$ plasma samples, there are good intra- and inter-day precision $(\mathrm{RSD}<5 \%, \mathrm{n}=3)$ respectively (Table I).
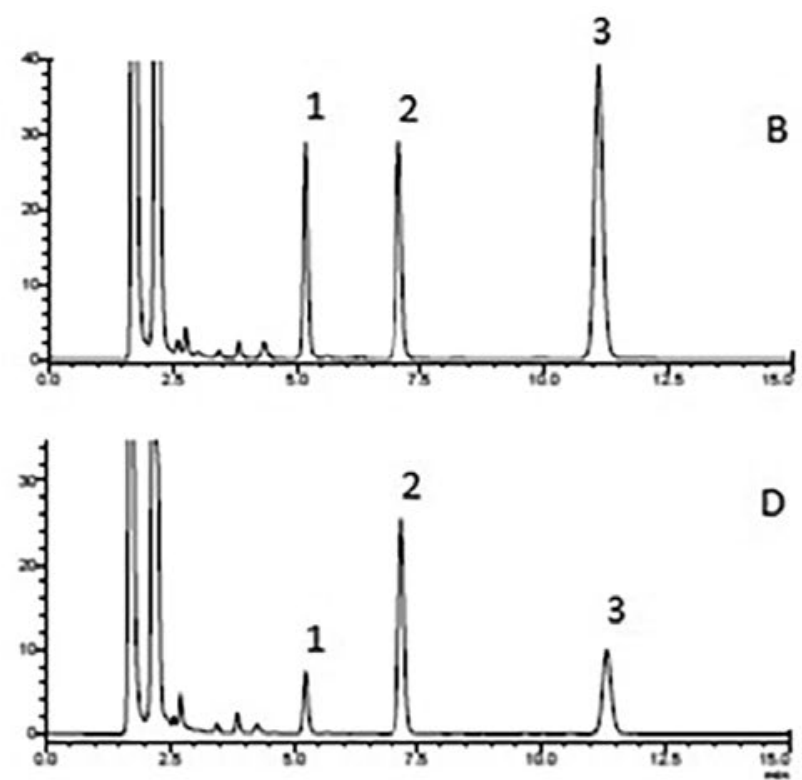

FIGURE 2 - HPLC profiles of diclofenac and its metabolite 4-hydroxy diclofenac in rats and in human recombinant CYP2C9 yeast cells. A. Blank plasma; B. Blank plasma spiked with reference substance; C. Sample before administration of diclofenac; D. Sample 15 min after administration of Diclofenac+Bifendate. 1. 4'-hydroxydiclofenac 2. Bifendate 3. Diclofenac. 
TABLE I - Intra and Inter-day precision of diclofenac and 4'-hydroxydiclofenac $(n=3)$

\begin{tabular}{lcccc}
\hline & $\begin{array}{c}\text { Sample concentration } \\
\left(\mathbf{C} / \boldsymbol{\mu} \cdot \mathbf{m L}^{-1}\right)\end{array}$ & $\begin{array}{c}\text { Mean concentration } \pm \\
\mathbf{S D}\left(\mathbf{C} / \mathbf{\mu g} \cdot \mathbf{m L}^{-1}\right)\end{array}$ & Intra-day RSD/\% & Inter-day RSD/\% \\
\hline \multirow{3}{*}{ Diclofenac } & 0.5 & $0.466 \pm 0.015$ & 3.19 & 3.19 \\
& 5 & $5.05 \pm 0.12$ & 2.46 & 0.83 \\
& 40 & $39.78 \pm 0.14$ & 0.35 & 0.60 \\
\hline \multirow{3}{*}{ ''-hydroxydiclofenac $^{-}$} & 0.5 & $0.469 \pm 0.020$ & 3.19 & 4.35 \\
& 5 & $5.10 \pm 0.10$ & 1.90 & 0.45 \\
& 40 & $39.85 \pm 0.21$ & 0.52 & 0.64 \\
\hline
\end{tabular}

\section{Effect of iguratimod on the pharmacokinetics of diclofenac and $4^{\prime}$-hydroxy diclofenac in rats}

The plasma concentration-time profile for diclofenac and 4'-hydroxy diclofenac given with IGU at low and high dose are shown in Figure 1. The pharmacokinetic parameters such as area under the curve (AUC), half-life $\left(\mathrm{T}_{1 / 2}\right)$, volume of distribution $(\mathrm{Vz})$ clearance $(\mathrm{CL})$ for diclofenac and 4 '-hydroxy diclofenac at control, low and high dose are summarized in Table I and II for in vivo study. The PK parameters of diclofenac plasma concentration when co-administered with IGU at low and high dose does not show any considerable changes associated with the control $(P>0.05$, Table II). Similarly, at low dose IGU and 4 '-hydroxy diclofenac does not show any significant differences compared with control $(p>0.05)$. However, the 4 '-hydroxy diclofenac showed significant differences at the high dose of IGU compared with control $(p<0.001)$.

In this study, the mean $\mathrm{T}_{1 / 2}$ for $4^{\prime}$-hydroxy diclofenac significantly increased from $13.56 \pm 7.05$ to $28.84 \pm 15.95$ $(p>0.05)$; mean $\mathrm{AUC}_{0-24}$ increased from $9.92 \pm 2.78$ to $47.14 \pm 11.75(p<0.001)$; mean CL $(\mathrm{mL} / \mathrm{h} / \mathrm{kg})$ decreased from $1.17 \pm 0.35$ to $0.16 \pm 0.06(p<0.001)$; also $\mathrm{Vz}(\mathrm{ml} / \mathrm{kg})$ decreased from $20.98 \pm 7.96$ to $5.95 \pm 1.67(p<0.01)$ at high dose i.e., $30 \mathrm{mg} / \mathrm{kg}$. (Table III)

\section{Effect of iguratimod on the metabolism of diclofenac in recombinant human $\mathrm{CYP2C} 9$ yeast cells}

The in vitro study was carry out in recombinant human CYP2C9 yeast cell system. There was no significant interaction at low and medium doses of IGU $(p>0.05)$. However, the high dose of IGU significantly decreased in an inhibitory response rate of diclofenac from $32.33 \pm 8.58 \%$ to $12.01 \pm 4.77 \%$ ( $p<0.001$, Table IV).

\section{DISCUSSION}

Generally, there are two types of drug interaction studies such as pharmacokinetics (PK) and pharmacodynamics (PD). Between PK interactions, the CYPs in the gut wall and liver, as well as the drug carrier, i.e. P-glycoprotein plays a major part, however also other enzymes and transport systems might be concerned. CYP enzymes are essential for the clearance of distant substances and breakdown of drugs. Nearly there are more than 50 CYP enzymes, but the CYP1A2, CYP2C9, CYP2C19, CYP2D6, CYP3A4, and CYP3A5 enzymes metabolize $90 \%$ of drugs available in the market. These enzymes primarily expressed in the liver, they additionally

TABLE II - Comparison of pharmacokinetics parameters of diclofenac in the presence of iguratimod

\begin{tabular}{lccc}
\hline Parameters & Control DA & Low Dose & High Dose \\
& & IG $(\mathbf{1 0} \mathbf{~ m g} / \mathbf{k g})+\mathbf{D A}$ & IG (30 $\mathbf{~ m g} / \mathbf{k g})+\mathbf{D A}$ \\
\hline $\mathrm{T}_{1 / 2}$ & $5.81 \pm 1.56$ & $7.79 \pm 4.37$ & $9.92 \pm 4.20$ \\
$\mathrm{AUC}_{0 \rightarrow 24 \mathrm{~h}}$ & $35.08 \pm 9.3$ & $32.66 \pm 5.73$ & $43.80 \pm 10.95$ \\
$\mathrm{CL}(\mathrm{mL} / \mathrm{h} / \mathrm{kg})$ & $0.43 \pm 0.11$ & $0.44 \pm 0.08$ & $0.33 \pm 0.10$ \\
$\mathrm{Vz}(\mathrm{mL} / \mathrm{kg})$ & $3.64 \pm 1.43$ & $4.77 \pm 2.37$ & $4.57 \pm 1.65$ \\
\hline
\end{tabular}

DA, diclofenac; IGU, iguratimod; T1/2, half-life; $\mathrm{AUC}_{0 \rightarrow 24 \mathrm{~h}}$, area under the curve; CL, clearance; Vz, the volume of distribution. All values are Mean $\pm \mathrm{SD} .{ }^{* * *} p<0.001,{ }^{* *} p<0.01,{ }^{*} p<0.05$ when compared to the normal saline group (one way ANOVA followed by Games Howell test to compare to each column to column) 
TABLE III - Comparison of pharmacokinetics parameters of 4 hydroxy diclofenac in the presence of iguratimod

\begin{tabular}{lccc}
\hline Parameters & Control OHDA & Low Dose & High Dose \\
& & IG $(10 \mathrm{mg} / \mathrm{kg})+\mathrm{OHDA}$ & $\mathrm{IG}(30 \mathrm{mg} / \mathrm{kg})+\mathrm{OHDA}$ \\
\hline $\mathrm{T}_{1 / 2}$ & $13.56 \pm 7.05$ & $12.05 \pm 3.81$ & $28.84 \pm 15.95$ \\
$\mathrm{AUC}$ & $9.92 \pm 2.78$ & $13.12 \pm 2.62$ & $47.14 \pm 11.75^{* * *}$ \\
$\mathrm{CL}(\mathrm{mL} / \mathrm{h} / \mathrm{kg})$ & $1.17 \pm 0.35$ & $0.94 \pm 0.27$ & $0.16 \pm 0.06^{* * *}$ \\
$\mathrm{Vz}(\mathrm{mL} / \mathrm{kg})$ & $20.98 \pm 7.96$ & $15.79 \pm 4.95$ & $5.95 \pm 1.67^{* *}$ \\
\hline
\end{tabular}

DA, diclofenac; IGU, iguratimod; OHDA, 4-hydroxy diclofenac; T1/2, half-life; $\mathrm{AUC}_{0 \rightarrow 24 \mathrm{~h}}$, area under the curve; CL, clearance; $\mathrm{Vz}$, the volume of distribution. All values are $\mathrm{Mean} \pm \mathrm{SD}(\mathrm{n}=8) .{ }^{* * *} p<0.001,{ }^{* *} p<0.01,{ }^{*} p<0.05$ when compared to the normal saline group (one way ANOVA followed by Games Howell test to compare to each column to column)

TABLE IV - Inhibitory Effect of Iguratimod on Diclofenac metabolism in human recombinant CYP2C9 yeast cells

\begin{tabular}{lcccc}
\hline Groups & Control group & $\begin{array}{c}\text { Low Dose group } \\
(\mathbf{0 . 2 5} \mathbf{~ m M / L )}\end{array}$ & $\begin{array}{c}\text { Medium dose group } \\
(\mathbf{0 . 5} \mathbf{~ m M} / \mathbf{L})\end{array}$ & $\begin{array}{c}\text { High Dose group } \\
(\mathbf{1 ~ m M} / \mathbf{L})\end{array}$ \\
\hline Inhibitory response rate (\%) & $32.33 \pm 8.58$ & $37.80 \pm 5.96$ & $33.94 \pm 5.28$ & $12.01 \pm 4.77^{* * *}$ \\
\hline
\end{tabular}

All values are Mean $\pm \mathrm{SD}(\mathrm{n}=8)$. DA: diclofenac. ${ }^{* * *} p<0.001,{ }^{* *} p<0.01,{ }^{*} p<0.05$ when compared to the normal saline group (one way ANOVA followed by Games Howell test to compare to each column to column)

appear in the kidneys, small intestine, placenta and lungs (Slaughter, Edwards, 1995; Wilkinson, 2005).

In the current study, we examined and analysed the effect of IGU on the pharmacokinetics of diclofenac and 4 '-hydroxy diclofenac in rats. Furthermore, we also examined the inhibitory effects of IGU on diclofenac in recombinant human CYP2C9 yeast cell system. Our investigation showed that there was no significant interaction between low dose IGU and diclofenac both in vivo and in vitro studies $(p>0.05)$. However, at higher doses i.e. $30 \mathrm{mg} / \mathrm{kg}$ IGU significantly increased the plasma concentration of 4'-hydroxy diclofenac but did not increase diclofenac plasma concentration as compared with the control group in rats (Table III, Figure 2). Moreover, at high dose, IGU significantly inhibited the metabolism of diclofenac in vitro (Table IV). These results give a possible direction that IGU may not directly show an inhibitory effect on the activity of CYP2C9 in vivo. However, we suggest that IGU or its metabolites might show inhibitory effect on glucuronidation of 4 '-hydroxy diclofenac to form 4 '-hydroxy diclofenac glucuronide.

Toyama Chemical Co., Ltd. has conducted inhibition studies on metabolites of IGU previously, the metabolites of IGU, i.e., M1, M2, M3, M4, and M5, at a dilution of $100 \mu \mathrm{M}$ inhibited tolbutamide 4-hydroxylase action of HLMs by $34.3 \%, 44.9 \%, 19.3 \%, 29.3 \%$, and $29.1 \%$, correspondingly. Between these metabolites, M4 is formed precisely from IGU by numerous CYP isoforms, including CYP2C9. These findings suggest that IGU metabolites, such as M2, which demonstrate a noteworthy inhibitory effect on CYP2C9 action, is equivalent to that of IGU (Taisho Toyama Pharmaceutical Co., 2013). As the maximum complete plasma concentration of $\mathrm{M} 2$ regarding frequent oral administration of IGU is nearly 2- folds greater than that of IGU (Taisho Toyama Pharmaceutical Co., 2013). It is likely that M2 as well as IGU play an essential part in inhibition of CYP2C9-facilitated diclofenac metabolism, which might reinforce by our outcomes of our in vitro study. However, there is no direct indication that IGU metabolites, including M2, inhibit diclofenac metabolism. Advanced studies are needed to describe the inhibitory effects of IGU metabolites. Our results indicate that at high dosage of IGU the mean $\mathrm{T}_{1 / 2}$ of $4^{\prime}$-hydroxy diclofenac increased by $212 \%(p>0.05)$, increase the mean AUC by $475 \%(\mathrm{P}<0.001)$, and decrease clearance by $13 \%(p<0.001)$, with the control group in the in vivo study. These results suggest that IGU or its metabolites may play an inhibitory effect on UGT enzyme that metabolizes the $4^{\prime}$-hydroxy diclofenac to $4^{\prime}$-hydroxy diclofenac glucuronide.

Diclofenac is an efficient and commonly approved NSAIDS, that is metabolized by numerous drug metabolizing routes, including cytochrome P450s (CYPs) and uridine 5-diphosphoglucuronosyl transferase (UGTs) (King et al., 2001). The general metabolic route of diclofenac to form 4-hydroxy diclofenac is catalyzed primarily by CYP2C9, which is distinguished to reveal a naturally controlled polymorphism causing in changed 
pharmacokinetic and pharmacodynamic properties of certain clinically significant drugs (Yu et al., 2005). While human UGT2B7 and rat UGT2B1 is the main enzyme concerned in catalyzing the glucuronidation of diclofenac in HLMs and rats (Wei, 2003). The in vitro inhibitory effect of IGU was studied in HLMs, as explained in an article issued by the agency of health, labor and safety Japan, that IGU at a concentration of $100 \mu \mathrm{M}$ inhibits CYP2C9 action by 50\% (Ruddy, Austen, 1975). In our in vitro study, high doses of IGU significantly inhibited the diclofenac metabolism in the recombinant human CYP2C9 yeast cell culture system, its metabolism rate is decreased by $37 \%$ compared with the control phase (Table III). Our data estimated that diclofenac is metabolized by CYP2C9, and IGU inhibits the CYP2C9 activity in recombinant human
CYP2C9 yeast cells. The hypothesis described above have suggested predominantly on in vitro study in which oxidative metabolism is the single choice existing for the substrate, while various pathways may subsist in in vivo for metabolism of diclofenac in the liver.

Several CYP2C9 inhibitors exhibit substratedependent inhibitory effects (Hanatani et al., 2003; Kumar et al., 2006). Kumar et al. (2006) observed the inhibitory relationship of 28 complexes with five often functioned in vitro probe substrates for CYP2C9. Diclofenac used as the comparator (it is a probe for CY92C9 most frequently used), once the inhibition of every compound competed for the additional substrates, inhibitors of (S)-warfarin metabolism indicated the highest amount of variations arrangement in both CYP2C9.1 and CYP2C9.3 enzymes.
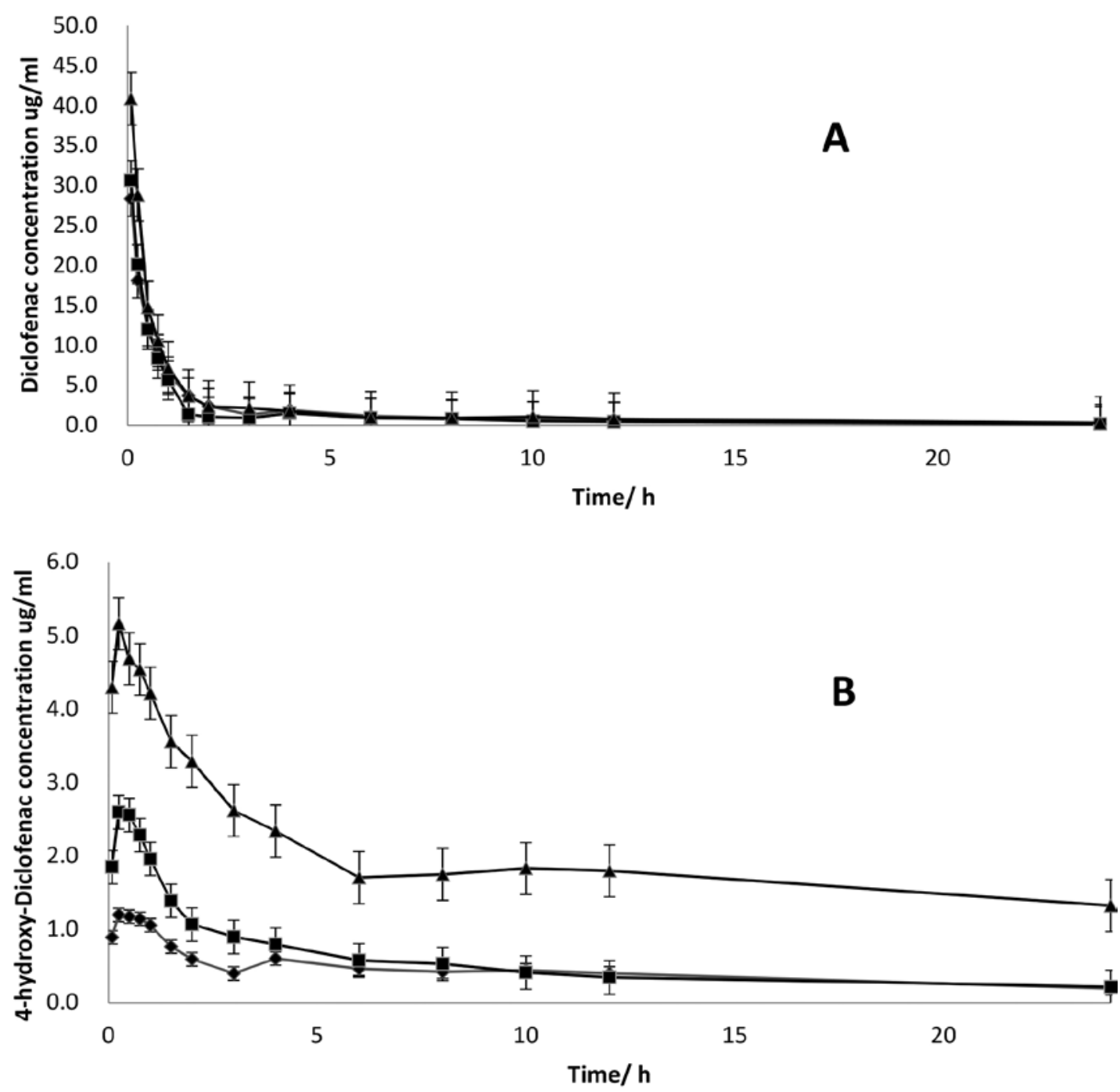

FIGURE 3 - Mean plasma concentration-time profile of diclofenac and 4'-hydroxy diclofenac with iguratimod in rats. The concentration of diclofenac (A) and 4-hydroxy diclofenac (B) in plasma were plotted against the time after administration of $15 \mathrm{mg} / \mathrm{kg}$ diclofenac and IGU at normal saline $(\boldsymbol{\square})$, low IGU $10 \mathrm{mg} / \mathrm{kg}(\bullet)$ and high IGU $30 \mathrm{mg} / \mathrm{kg}(\boldsymbol{\Delta})$. Each value represents the mean \pm S.D. of eight animals each. (A) Diclofenac (B) 4'-hydroxydiclofenac. 
Twelve inhibitors of CYP2C9.1 and ten inhibitors of CYP2C9.3 when (S) warfarin was used as a substrate probe turn out to be considered as ensuing of more interest. Eight inhibitors of the 12 in CYP2C9.1 and four inhibitors of CYP2C9.3 shifted from the average of the highest concern category, as matched with when diclofenac was exploited as the probe. Thus, the substrate probes dropped into three separate divisions varying in genotype, proposing that several probes could be required to completely evaluate inhibition of CYP2C9 in vitro. A pattern of inhibition studies using diclofenac and (S)warfarin might give the widest 'biochemical space' in particular to CYP2C9 inhibition (Kumar et al., 2006).

\section{CONCLUSION}

The results demonstrate that IGU at low dose shows no inhibitory effect on the pharmacokinetic parameters of diclofenac and 4-hydroxy diclofenac in rats; also at a low dose, it shows no inhibitory effect on the metabolism of diclofenac in human recombinant CYP2C9 yeast cells. However, at high dose, IGU shows a major variation in pharmacokinetics parameters of 4'-hydroxy diclofenac in rats, as well as it shows a direct inhibition of diclofenac metabolism in human recombinant CYP2C9 yeast cells. Beside this, our study also gives a possible direction that IGU or its metabolites may inhibit UGT mediated metabolism of 4 '-hydroxy diclofenac to form 4 '-hydroxy diclofenac glucuronide. Nevertheless, mechanism based inhibition of UGT enzyme by IGU metabolites and wellcontrolled scientific studies needed to verify the effect of IGU or its metabolites on CYP2C9 and UGT.

\section{CONFLICT OF INTEREST}

The authors proclaim no conflict of interest.

\section{ACKNOWLEDGEMENTS}

This study was supported by the Program for Jiangsu Province "Shuang Chuang" Team (to YPANG Chang Qing).

\section{REFERENCES}

Bort R, Macé K, Boobis A, Gómez-Lechón MJ, Pfeifer A, Castell J. Hepatic metabolism of diclofenac: role of human CYP in the minor oxidative pathways. Biochem Pharmacol. 1999;58(5):787-796.
Goldstein JA, de Morais SM. Biochemistry and molecular biology of the human CYP2C subfamily. Pharmacogenetics. 1994;4(6):285-299.

Hanatani T, Fukuda T, Onishi S, Funae Y, Azuma J. No major difference in inhibitory susceptibility between CYP2C9.1 and CYP2C9.3. Eur J Clin Pharmacol. 2003;59(3):233-235.

Hara M, Ishiguro N, Katayama K, Kondo M, Sumida T, Mimori $\mathrm{T}$, et al. Safety and efficacy of combination therapy of iguratimod with methotrexate for patients with active rheumatoid arthritis with an inadequate response to methotrexate: An open-label extension of a randomized, double-blind, placebo-controlled trial. Mod Rheumatol. 2014;24(3):410-418.

Inoue K, Yamazaki H, Imiya K, Akasaka S, Guengerich FP, Shimada T. Relationship between CYP2C9 and 2C19 genotypes and tolbutamide methyl hydroxylation and S-mephenytoin 4'-hydroxylation activities in livers of Japanese and Caucasian populations. Pharmacogenetics. 1997;7(2):103-113.

Ishiguro N, Yamamoto K, Katayama K, Kondo M, Sumida T, Mimori T, et al. Concomitant iguratimod therapy in patients with active rheumatoid arthritis despite stable doses of methotrexate: a randomized, double-blind, placebo-controlled trial. Mod. Rheumatol. 2013;23(3):430-439.

King C, Tang W, Ngui J, Tephly T, Braun M. Characterization of rat and human UDP-glucuronosyltransferases responsible for the in vitro glucuronidation of diclofenac. Toxicol Sci. 2001;61(1):49-53.

Kumar V, Wahlstrom JL, Rock DA, Warren CJ, Gorman LA, Tracy TS. CYP2C9 inhibition: impact of probe selection and pharmacogenetics on in vitro inhibition profiles. Drug Metab Dispos. 2006;34(12):1966-1975.

Leemann T, Transon C, Dayer P. Cytochrome P450TB (CYP2C): A major monooxygenase catalyzing diclofenac 4'-hydroxylation in human liver. Life Sci. 1993;52(1):29-34.

Mancy A, Antignac M, Minoletti C, Dijols S, Mouries V, Duong NT, et al. Diclofenac and its derivatives as tools for studying human cytochromes $\mathrm{P} 450$ active sites: particular efficiency and regioselectivity of P450 2Cs. Biochemistry. 1999;38(43):1426414270.

Miners JO, Birkett DJ. Cytochrome P4502C9: an enzyme of major importance in human drug metabolism. Br J Clin Pharmacol. 1998;45(6):525-538. 
Ruddy S, Austen KF. Activation of the complement and properdin systems in rheumatoid arthritis. Ann N Y Acad Sci. 1975;256:96-104.

Slaughter RL, Edwards DJ. Recent advances: the cytochrome P450 enzymes. Ann Pharmacother. 1995;29(6):619-624.

Small RE. Diclofenac sodium. Clin Pharm. 1989;8(8):545-558.

Taisho Toyama Pharmaceutical Co., L. Interview form (KOLBET® Tablets 25 mg). 6th ed. Japan; 2013

Tracy TS, Marra C, Wrighton SA, Gonzalez FJ, Korzekwa KR. Studies of flurbiprofen 4'-hydroxylation. Additional evidence suggesting the sole involvement of cytochrome P450 2C9. Biochem Pharmacol. 1996;52(8):1305-1309.

Wei T. The Metabolism of diclofenac - enzymology and toxicology perspectives. Curr Drug Metab. 2003;4(4):319-329.

Wilkinson GR. Drug metabolism and variability among patients in drug response. New England J Med. 2005;352(21):22112221.
Yamaori S, Takami K, Shiozawa A, Sakuyama K, Matsuzawa $\mathrm{N}$, Ohmori S. In vitro inhibition of CYP2C9-mediated warfarin 7-hydroxylation by iguratimod: possible mechanism of iguratimod-warfarin interaction. Biol Pharm Bull. 2015;38(3):441-447.

Yamazaki H, Inoue K, Chiba K, Ozawa N, Kawai T, Suzuki Y, et al. Comparative studies on the catalytic roles of cytochrome P450 2C9 and its Cys- and Leu-variants in the oxidation of warfarin, flurbiprofen, and diclofenac by human liver microsomes. Biochem Pharmacol. 1998;56(2):243-251.

Yu LJ, Chen Y, Deninno MP, O'Connell TN, Hop CE. Identification of a novel glutathione adduct of diclofenac, 4'-hydroxy-2'-glutathion-deschloro-diclofenac, upon incubation with human liver microsomes. Drug Metab Dispos. 2005;33(4):484-488.

Received for publication on $11^{\text {th }}$ May 2017 Accepted for publication on $19^{\text {th }}$ April 2018 